\title{
Selected tumour biomarker levels in sheep with pulmonary adenomatosis
}

\author{
Cumali Özkan ${ }^{1}$, Serkan Yıldırım², Zübeyir Huyut ${ }^{3}$, Mustafa Özbek ${ }^{1 凶}$ \\ ${ }^{1}$ Department of Internal Medicine, Faculty of Veterinary Medicine, Van Yüzüncü Y1l University, 65080, Van, Turkey \\ ${ }^{2}$ Department of Pathology, Faculty of Veterinary Medicine, Atatürk University, 25240, Erzurum, Turkey \\ ${ }^{3}$ Department of Biochemistry, Faculty of Medicine, Van Yüzüncü Yıl University, 65080, Van, Turkey \\ mozbek@dr.com
}

Received: July 7, 2019 Accepted: February 21, 2020

\begin{abstract}
Introduction: Sheep pulmonary adenomatosis (ovine pulmonary adenomatosis, OPA, Jaagsiekte) is a chronic contagious bronchoalveolar carcinoma caused by the Jaagsiekte sheep retrovirus. Since effective treatment and a vaccination procedure are not currently possible, control and eradication of the disease is difficult. It leads to serious economic losses around the world, therefore studies are currently underway in order to design control and eradication programmes. In this study, levels and changes in selected tumour markers (carcinoembryonic antigen (CEA), carbohydrate antigen (CA) 125, CA 19-9, CA 15-3, and alphafetoprotein (AFP)-3) and their diagnostic significance were investigated. Material and Methods: A total of 30 sheep were used. Clinical examinations were performed and blood samples were obtained before slaughter from all animals with presumed OPA. Blood samples with positive OPA results by macroscopic and histopathological examination were included in the study as the experimental group and numbered 20. Sheep totalling 10 had negative OPA results and provided control samples. Results: CEA levels were similar in both groups, and the differences were statistically insignificant ( $P>0.05)$. CA 125, CA 19-9, CA 15-3, and AFP-3 levels were higher in the OPA group than the control group and with statistical significance $(\mathrm{P}<0.05)$. In all OPA animals, CA 125 levels were higher than $1 \mathrm{U} / \mathrm{mL}$. Conclusion: Serum CAs and AFP levels increase significantly in adenomatous sheep. These tumour markers are thought to facilitate the diagnosis of OPA.
\end{abstract}

Keywords: sheep, pulmonary adenomatosis, tumour biomarkers, diagnosis.

\section{Introduction}

Sheep pulmonary adenomatosis (ovine pulmonary adenomatosis, OPA, Jaagsiekte) is a chronic contagious bronchoalveolar carcinoma caused by Jaagsiekte sheep retrovirus (JSRV) $(9,20)$. Infection occurs in all sheep breeds and may also occasionally affect goats. In infected goats, kids, and lambs, the disease usually follows a subclinical course $(18,32)$. The disease was first described in South Africa in 1865, and now a century and a half later it is currently emerging across much of the world and inflicting grave economic losses in the sheep industry in several regions, New Zealand, Australia, and Iceland being the only unaffected countries $(4,9,12)$. The disease caused losses up to $50-80 \%$ in the countries where it first emerged, and its incidence is $1-2 \%$ in endemic regions $(1,17,18)$.

Anamnesis, clinical findings, necropsy, histopathology, immunohistochemistry, electron microscopy, polymerase chain reaction (PCR), ultrasonography, and computed tomography may be helpful in the diagnosis of the disease $(9,12)$. Detecting JSRV proteins may be helpful for laboratory diagnosis; however, specific JSRV antibodies are not present in blood, and these proteins can only be detected within a tumoural mass in the lungs of sick animals. Therefore, there is no existing laboratory test for diagnosing the disease in live animals (1). In adult sheep, chronic pneumonia that does not respond to antibiotic treatment and proves fatal may suggest this disease. However, the definitive diagnosis can only be made by necropsy and histopathological examination $(2,5,9,11,22)$. PCR, even with a high quotient of false-negative results, may be used to detect infected herds for epidemiological studies but is not suitable to detect the disease individually for eradication purposes. Ultrasonography is an alternative and more accurate diagnostic method and can be used for detecting large tumours in animals with subclinical infections. Therefore, infected animals can be detected in the early stages and separated from the herds. Computed 
tomography has also been reported for diagnosing OPA; however, its cost prevents its widespread usage $(12,31)$.

Since there is not any effective treatment or vaccination, control and eradication of the disease is difficult. OPA leads to considerable economic losses in several regions of the world, therefore studies are currently in progress in order to establish control and eradication programmes. Natural infection occurs in sheep by the aerosol route, whereas in younger animals, it occurs by colostrum and milk ingestion $(1,12,29,31)$.

OPA and human lung adenocarcinomas have similar clinical, morphological, pathological, and histopathological features, and consequently it is reported that OPA can form a decent model for human lung adenocarcinomas $(5,12,20,33,40)$. Tumour markers have been used in human medicine for scanning, diagnosis, prognosis, and monitoring cancer cases. Markers such as carcinoembryonic antigen (CEA), cytokeratin fragment (CYFRA 21-1), and cancer or carbohydrate antigens (CA) 15-3, 19-9, and 125 have been widely used for diagnosing and tracking treatment of various tumour types $(24,25)$. No ideal tumour marker exists; however, markers such as CEA, CAs, CYFRA 21-1, and $\alpha$-fetoprotein (AFP) have been used for monitoring several cancer patients (10). In our literature searches, we did not find any study concerning the evaluation of CEA, CA 125, CA 19-9, CA 15-3, and AFP-3 levels in sheep with OPA.

In this study, the levels and changes in these tumour markers used in human medicine as well as the significance of these markers in diagnosis of the disease were investigated.

\section{Material and Methods}

The animal material consisted of sheep two years old and older brought to a private slaughterhouse. Before slaughter, blood samples were obtained from all animals that were clinically examined and presumed to be infected with pulmonary adenomatosis. Following slaughter, blood samples of animals which were OPApositive according to macroscopic and histopathological examinations were included in the study. Blood samples obtained from a total of 30 sheep - 20 sheep with positive and 10 with negative OPA results - were used in this study. Each sample was collected from the jugular vein partly into a tube with EDTA and partly into a coagulant-free tube. Samples in coagulant-free tubes were centrifuged at $3,000 \times \mathrm{g}$ for $10 \mathrm{~min}$ (Rotofix 32,
Hettich, Tuttlingen, Germany) and serum samples were extracted. Extracted sera were stored at $-20^{\circ} \mathrm{C}$ until the assay was performed. Concentrations of tumour markers such as CEA, CA 125, CA 19-9, CA 15-3, and AFP-3 were measured in an autoanalyser (Architect System i4000SR, Abbott, Abbott Park, IL, USA) by chemiluminescent microparticle immunoassay (CMIA) method with the calibrator and control and test kits dedicated to the device.

Macroscopic and histopathological examinations were performed on the lungs of the OPA-suspected animals. Following macroscopic examinations, lung tissues were obtained from the sick animals and fixed in $10 \%$ formalin solution for $48 \mathrm{~h}$ and washed under flowing tap water for $10 \mathrm{~h}$. Tissue samples were passed through a graded series of alcohol and xylol, and $4 \mu \mathrm{m}$ thick sections were prepared on microscope slides. Preparations were stained by haematoxylin-eosin (HE) and examined under a light microscope (DM 1000, Leica, Wetzlar, Germany).

Statistical analysis. Descriptive statistical values were given as mean and standard deviation for continuous variables. Student's $t$-test was used for group comparison following variant analysis. The statistical significance level was accepted as $5 \%(\mathrm{P}<0.05)$ and SPSS 20.0 (IBM SPSS Inc., Armonk, NY, USA) software was used for statistical calculations.

\section{Results}

Clinical findings. Clinical examinations showed most of the animals to have dyspnoea, and this condition worsened when the animals were forced to walk. A frothy exudate flowed from the nose when the hind legs of the animals were raised. Loss of appetite, coughing, and weight loss were also observed in some animals. Serious sound changes were not heard in lung auscultation in any but a few animals. In addition to these findings, increases in body temperature and heart and respiratory rates were detected.

Biochemical findings. Tumour biomarkers belonging to OPA and control group animals are quantified in Table 1. CEA levels were similar in animals in the OPA and control groups, and the differences were statistically insignificant $(P>0.05)$. However, CA 125, CA-19, CA 15-3, and AFP-3 concentrations in animals with OPA were significantly higher than those in the control group $(\mathrm{P}<0.05)$. CA 125 concentrations, particularly, were higher than $1 \mathrm{U} / \mathrm{mL}$ in all animals with OPA, and this result was remarkable.

Table 1. Tumour biomarker results in healthy and OPA sheep

\begin{tabular}{lll}
\hline Tumor biomarker & Control $(\overline{\mathrm{x}} \pm \mathrm{S} \overline{\mathrm{x}})$ & OPA $(\overline{\mathrm{x}} \pm \mathrm{S} \overline{\mathrm{x}})$ \\
\hline CEA $(\mathrm{ng} / \mathrm{mL})$ & $<0.5$ & $<0.5$ \\
CA $19-9(\mathrm{U} / \mathrm{mL})$ & $1.495 \pm 0.104$ & $1.759 \pm 0.151^{*}$ \\
CA $15-3(\mathrm{U} / \mathrm{mL})$ & $1.028 \pm 0.146$ & $1.481 \pm 0.156^{*}$ \\
CA $125(\mathrm{U} / \mathrm{mL})$ & $<1$ & $1.235 \pm 0.211^{*}$ \\
AFP-3 $(\mathrm{ng} / \mathrm{mL})$ & 0.001 & $0.038 \pm 0.017^{*}$ \\
\hline
\end{tabular}

* Means in the same row with asterisks are statistically significant $(\mathrm{P}<0.05)$ 

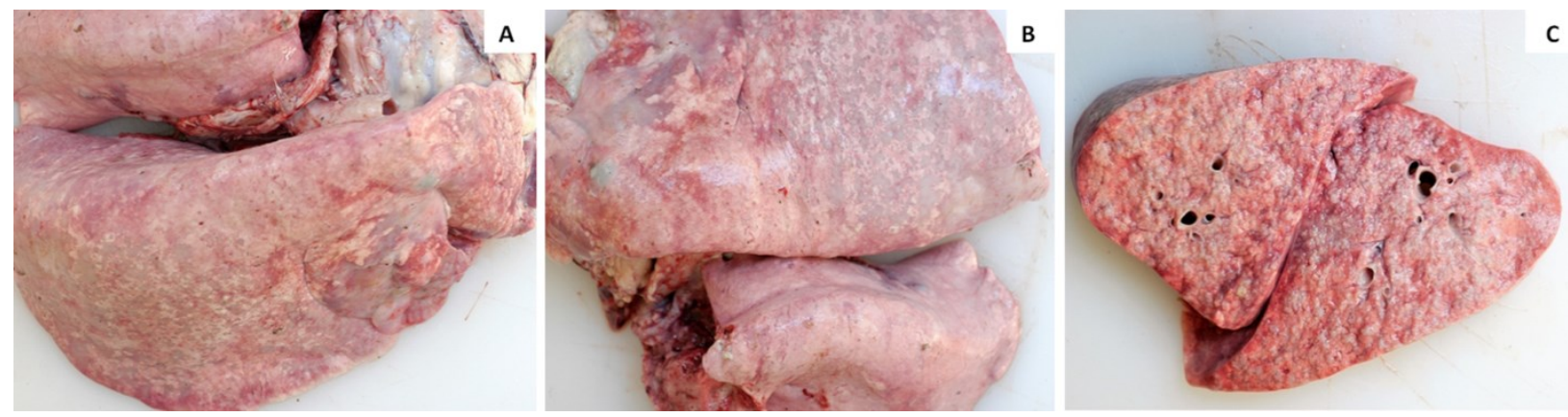

Fig. 1. Macroscopic appearance of the lungs. A - grey-white tumoural foci on a diaphragmatic lung lobe; B - large foci consisting of merged small foci; $\mathrm{C}$ - effusive greyish tumoural foci in cross-section of the lung

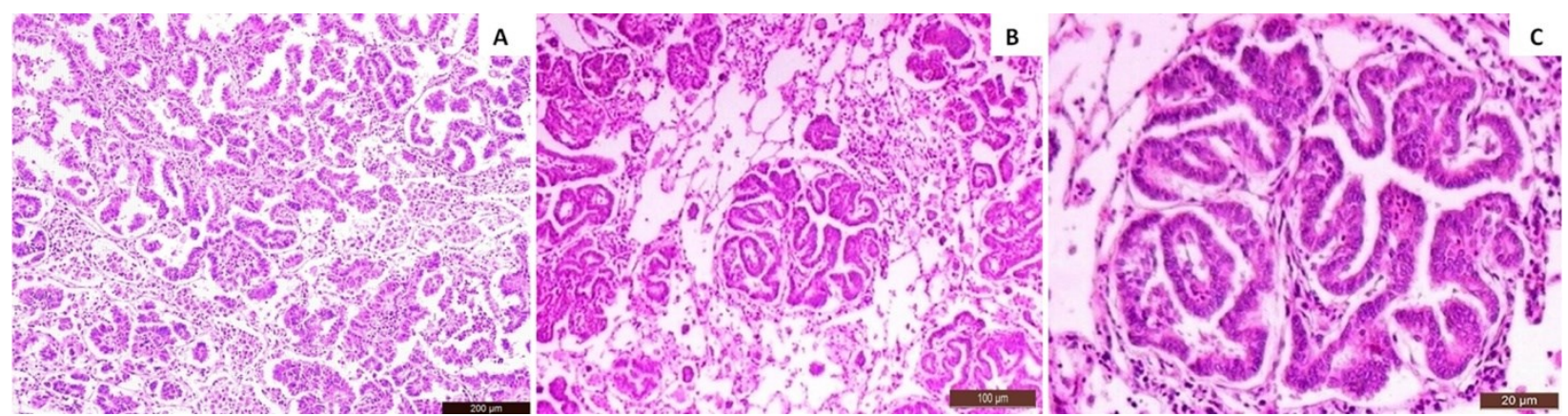

Fig. 2. Microscopic appearance of the lungs. A - type-II pneumocytes proliferating in alveolar lumen and numerous macrophages on adjacent alveoli; H\&E, Bar: $200 \mu \mathrm{m}$; B - type-II pneumocytes proliferating in alveolar lumen and numerous macrophages on adjacent alveoli; H\&E, Bar: $100 \mu \mathrm{m} ; \mathrm{C}$ - type-II pneumocyte cells proliferating in alveolar lumens; H\&E, Bar: $20 \mu \mathrm{m}$

Pathological findings. Following necropsy, greywhite solid foci were observed in the lungs of animals presumed to have OPA. These foci were nodular and between lentil- and walnut-sized (Figs 1A and 1B). The lungs were enlarged, heavier than usual, and not collapsed. Additionally, large volumes of frothy exudate were present in lung sections. These lesions were effusive and grey in colour and commonly located at the diaphragmatic lung lobes (Fig. 1C).

Microscopic examinations showed that the type-II pneumocytes on the alveolar wall had papillary extensions towards the alveolar lumen, and that the Clara cells on the bronchiolar wall proliferated towards the lumen (Fig. 2A). In addition, adjacent alveoli were filled with macrophages (Figs $2 \mathrm{~A}$ and 2B). These anaplastic cells which had proliferated on the walls of the alveoli, bronchi, and bronchioles were poor in chromatin, were eosinophilic, and had vacuoles. Alveolar and bronchial lumens were narrowed due to cell proliferations (Fig. 2C).

\section{Discussion}

Since treatment for OPA does not exist, studies have been performed regarding diagnosis, prophylaxis, and eradication of the disease in recent years $(2,12,18)$. The absence of studies related to the levels and role of tumour markers in OPA directed us to perform such a study. In this research, the levels and changes in selected tumour markers used in human medicine as well as the contribution of these markers to diagnosis of the disease were investigated.

According to previous studies, the incubation period of the disease is long (six months to three years), therefore clinical symptoms usually develop in twoyear-old or older animals. However, clinical symptoms were also reported in animals less than one year of age, albeit rarely $(18,31,32)$. Clinical symptoms develop with the progression of tumoural mass in the lungs. The most typical clinical findings from observation of the disease were reported as severe dyspnoea and frothy, clear, milky, or sometimes pink fluid exuding from the nose when the hind legs of the animals were raised, using the technique called the "wheelbarrow test". The amount of fluid is usually $10-40 \mathrm{~mL}$, although it may reach $400 \mathrm{~mL}$ in some animals. It is also reported that sick animals may fall behind the herd and have exacerbated dyspnoea when forced to walk. Findings such as coughing and loss of appetite are not common; the appetite is usually normal. Severe loss of weight can be observed with the development of the disease. Auscultation of the lungs also does not assist in differential diagnosis of the disease. While pathological lung sounds cannot be heard, moist rales may be audible in some animals even without using a stethoscope. Death usually occurs due to secondary bacterial pneumonias and respiratory failure $(1,12,20,31,32)$.

In our study, most of the animals with OPA were two years of age or older and they were dyspnoeic. The dyspnoea was exacerbated when they were forced to walk, and some animals had severe frothy fluid exuding 
from their noses. Serious sound changes were not detected in most of the animals when their lungs were auscultated, however, an increase in respiratory rate was detected, as were rises in body temperature and heart rate. Some animals had coughing, weight loss, and anorexia. These clinical findings correspond with the previously reported clinical findings $(1,12,18,20,31,32)$.

Development of macroscopic lesions, particularly on the cranioventral lobes of the lungs, were attributed to the disease. These lesions were diffuse, solid, and merged nodules with grey-white colour resembling an icy glass. Histologically, the lesions were reported as generally consisting of proliferated foci in alveoli and were formed by cubic and prismatic epithelial cells proliferating towards the lumen of the alveoli and bronchioli. Some of these lesions were also reported to consist of acinar and solid structures (13, 14, 19, 27, 28). Additionally, it was also noted that developing tumour cells principally originate from type-II pneumocytes and the Clara cells $(9,18)$. With the emergence of a tumour, cancer cells occupy places in the normal structure of the lung and lead to both deterioration in normal lung function and excessive fluid secretion $(12,20,31)$. In this study, macroscopic and microscopic findings corresponded to these previously published observations.

OPA and human adenocarcinomas have similar morphological, pathological, and epidemiological features; thus, OPA can be used as a decent model for human lung adenocarcinomas $(12,20,33)$. In recent years, tumour biomarkers have gained importance and have been used for detection and management of various cancer types in human medicine. Tumour markers such as CEA, CYFRA 21-1, and CAs 15-3, 19-9, and 125 have been widely used for diagnosing and tracking treatment of various tumour types $(24,25)$. Additionally, combined usage of tumour biomarkers has great importance in diagnosis of cancer. An ideal tumour marker does not exist because each biomarker has own specificity and sensitivity. Nevertheless, tumour markers such as CEA, CAs, CYFRA 21-1, and AFP have been used for monitoring several cancer patients $(10,16,39,42)$. The main tumour markers used to diagnose lung cancer are CEA, NSE, and CYFRA 21-1. Nevertheless, cancer antigens such as CA 125 and CA 19-9 are present in high concentrations in lung cancer, and these markers were proposed as potentially exploitable for its detection $(15,34)$.

CEA is a membrane-associated glycoprotein that has functions such as cellular adhesion and signal transduction. It is secreted by several adenocarcinoma types including monoclonal CEA (mCEA) derivatives (26). CEA has been used for differential diagnosis of lung cancers as well as monitoring and detecting their recurrent conditions (15). Certain studies describe CEA as an important biomarker used in non-small cell lung cancer (NSCLC) for determining prognosis $(8,15)$. Tomita et al. (35) and Salgia et al. (30) detected an increase in CEA concentrations in stage III and IV
NSCLC patients, Chen et al. (6) reported high concentrations of CEA in benign lung lesions, and Zamay et al. (47) indicated a significant increase in CEA in patients with lung cancer. Although increases in CEA levels were reported in humans with lung cancer, there was not any change in CEA levels in sheep with OPA. In our study, CEA levels were lower than $0.5 \mathrm{ng} / \mathrm{mL}$ in both the control and OPA groups, and the differences were statistically insignificant. There is no existing method or kit for measuring CEA levels in OPA. Besides the non-existence of either a method or kit, a further problematic factor is that the testing suitability of sheep antibodies against CEA is unknown, since CEA has not been used for detecting cancer types in sheep and there is no information in the literature. In this study, the reason for not detecting any increase in CEA levels in OPA may be associated with the method of analysis, as it has been routinely used in human medicine for diagnosing various types of lung cancer.

CA 125 has been used as a tumour indicator for monitoring cancer patients and evaluating recurrence rates, especially for ovarian cancers. High serum CA 125 concentrations were reported in patients with ovarian cancers as well as in patients with liver disorders $(3,41)$. In addition, CA 125 has been commonly used in diagnosis of malignant gynaecological pelvic tumours. It is secreted in high amounts by epithelial ovarian cells and serous ovarian adenocarcinoma, and its concentrations increase when the disease progresses (7). Tuxen et al. (37) reported that in a large number of cases, the increase in CA 125 concentrations was parallel with the clinical progression of the disease. Besides in ovarian cancers, CA 125 concentrations also increased in endometrial, cervical, and lung cancers (3).

CA $19-9$ is an antigen associated with gastrointestinal cancer types that increases in patients with gastrointestinal carcinomas, particularly where the pancreas is affected (41). CA 19-9 is stated to be a useful biomarker for diagnosing gastrointestinal tract tumours, especially pancreatic and colorectal cancer, adenocarcinomas of ovarian pulpa and serous and mucous adenocarcinomas of the ovarium (7). Li et al. (21) and Wang et al. (38) discovered that serum CA 19-9 concentrations were higher in benign than in malignant lung tumours, but the increase was not adequately reliable for differential diagnosis. Tsoukalas et al. (36) reported that patients with abnormal serum CA 19-9 concentrations before an operation had lower survival rates than patients with normal concentrations. However, they also pointed out that using this evaluation is not an independent prognostic factor in multiple-variant survival analysis. In a study performed by Ma et al. (23) on patients with non-small-cell lung cancer, positivity rates for CA 125 and CA 19-9 were detected at 5\% and 4.9\%, respectively. Isaksson et al. (15) indicated that before an operation, the recurrence rate was high in patients with increased CA 19-9 and/or CA 125 concentrations.

CA $15-3$ is another tumour biomarker with a large cellular surface mucin glycoprotein that is secreted from 
several glandular and ductal epithelial cells as well as certain haematopoietic cells (41). Serum AFP has been used as a useful marker for recurrent hepatocellular carcinomas even before the clinical symptoms develop. High concentrations of serum AFP were reported in malignant tumours of the gastrointestinal tract, pancreas, lung, and kidney (41). In our study, CA 125, CA 19-9, CA 15-3, and AFP-3 concentrations were higher in sheep with OPA than in the control group and this increase was statistically significant. When these results are examined, it is apparent that an increase in these markers such as occurs in various human cancer types is also observable in animals with OPA.

As there was a significant increase in serum CAs and AFP, it is concluded that using the studied tumour biomarker panel may make an important contribution to the diagnosis of OPA. However, in further studies, it will be necessary to use more animals and assay the positivity rate of each tumour marker specifically. As a desirable result of this and subsequent research, these tumour markers may be proposed for use in routine diagnosis and control of OPA in sheep. Additionally, it is also concluded that performing further studies related to combined usage of different panels such as circulatory tumour markers, ctDNA, and micro RNA (miR-486, miR-30d, mir-1, etc.) might be useful.

Conflict of Interest Statement: The authors declare that there is no conflict of interests regarding the publication of this article.

Financial Disclosure Statement: The research was funded with the authors' own means.

Animal Rights Statement: Not applicable.

\section{References}

1. Amini F., Mostafa-Tehrani A.: A five-year survey (2002-2007) on ovine pulmonary adenomatosis in a mixed-breed sheep flock. Bulg J Vet Med 2013, 16, 139-142.

2. Azizi S., Tajbakhsh E., Fathi F.: Ovine pulmonary adenocarcinoma in slaughtered sheep: A pathological and polymerase chain reaction study. J S Afr Vet Assoc 2014, 85, $1-5$.

3. Bashizadeh-Fakhar H., Rezaie-Tavirani M., Zali H., Faraji R., Nejad E.K., Aghazadeh M.: The diagnostic value of serum CEA, CA-125, and ROMA Index in low-grade serous ovarian cancer. Int J Cancer 2018, 11, e63397.

4. Beytut E., Sözmen M., Erginsoy S.: Immunohistochemical detection of pulmonary surfactant proteins and retroviral antigens in the lungs of sheep with pulmonary adenomatosis. J Comp Pathol 2009, 140, 43-53.

5. Can-Sahna K., Eroksuz Y., Berber E., Sozdutmaz I.: Detection of exogenous Jaagsiekte sheep retrovirus in Turkey. Indian J Anim Sci 2015, 49, 498-502.

6. Chen F., Li J., Qi X., Qi J.: Diagnostic value of CYFRA 21-1 and carcinoembryonic antigen in diagnosis of operable lung cancer from benign lung disease. J Cancer Res Ther 2018, 14, 400-404.

7. Chen F., Shen J., Wang J., Cai P., Huang Y.: Clinical analysis of four serum tumor markers in 458 patients with ovarian tumors: diagnostic value of the combined use of HE4, CA125, CA19-9, and CEA in ovarian tumors. Cancer Manag Res 2018, 10, 1313-1318.

8. Crosbie P.A.J., Shah R., Summers Y., Dive C., Blackhall F.: Prognostic and predictive biomarkers in early stage NSCLC: CTCs and serum/plasma markers. Transl Lung Cancer Res 2013, 2, 382-397.

9. Dudek K., Lutnicki K., Bednarek D., Marczuk J., Kycko A., Reichert M.: Changes in blood parameters induced by experimental jaagsiekte sheep retrovirus infection. J Vet Res 2016, 60, 245-251.

10. Duffy M.J.: Tumor markers in clinical practice: a review focusing on common solid cancers. Med Prin Pract 2013, 22, 4-11.

11. Gonzalez L., Garcia-Goti M., Cousens C., Dewar P., Cortabarria N., Extramiana A.B., Ortin A., De las Heras M., Sharp J.M.: Jaagsiekte sheep retrovirus can be detected in the peripheral blood during the pre-clinical period of sheep pulmonary adenomatosis. J Gen Virol 2001, 82, 1355-1358.

12. Griffiths D.J., Martineau H.M., Cousens C.: Pathology and pathogenesis of ovine pulmonary adenocarcinoma. J Comp Pathol 2010, 142, 260-283.

13. Hod I., Herz A., Zimber A.: Pulmonary carcinoma (Jaagsiekte) of sheep. Ultrastructural study of early and advanced tumor lesions. Am J Pathol 1977, 86, 545-558.

14. Ilhan F., Vural S.A., Yildirim S., Sözdutmaz I., Alcigir M.E.: Expression of p53 protein, Jaagsiekte sheep retrovirus matrix protein, and surfactant protein in the lungs of sheep with pulmonary adenomatosis. J Vet Diagn Invest 2016, 28, 249-256.

15. Isaksson S., Jönsson P., Monsef N., Brunnstrom H., Bendahl P.O., Jonsson M., Staaf J., Planck M.: CA 19-9 and CA 125 as potential predictors of disease recurrence in resectable lung adenocarcinoma. PLoS One 2017, 12, e0186284.

16. Jiang Z.F., Wang M., Xuc J.L.: Thymidine kinase 1 combined with CEA, CYFRA21-1 and NSE improved its diagnostic value for lung cancer. Life Sci 2018, 194, 1-6.

17. Kiran M.M.: Pathological investigations on sheep pulmonary adenomatosis. Selcuk Univ J Vet Fac 1993, 9, 41-45.

18. Kıran M.M., Çiftçi M.K., Erer H., Ortatatlı M., Hatipoğlu F., Tuzcu M.: The diagnosis of sheep pulmonary adenomatosis by exfoliative cytology and bronchoalveolar lavage techniques and the comparison of results with histopathologic findings. Ataturk Univ J Vet Sci 2000, 16, 59-68.

19. Kycko A., Reichert M.: Overexpression of aldolase A and cytokeratin 19 in ovine pulmonary adenocarcinoma. Pol J Vet Sci $2012,15,703-709$

20. Leroux C., Girard N., Cottin V., Greenland T., Mornex J.F., Archer F.: Jaagsiekte sheep retrovirus (JSRV): from virus to lung cancer in sheep. Vet Res 2007, 38, 211-228.

21. Li X., Asmitananda T., Gao L., Gai D., Song Z., Zhang Y., Ren H., Yang T., Chen T., Chen M: Biomarkers in the lung cancer diagnosis: a clinical perspective. Neoplasma 2012, 59, 500-507.

22. Liu Y., Zhang Y.F., Sun X.L., Liu S.Y.: Detection of Jaagsiekte sheep retrovirus in the peripheral blood during the pre-clinical period of ovine pulmonary adenomatosis. Gen Mol Res 2016, 15, 8521 doi: $10.4238 / \mathrm{gmr} .15038521$

23. Ma S., Shen L., Qian N., Chen K.: The prognostic values of CA125, CA19.9, NSE, and SCC for stage I NSCLC are limited. Cancer Biomark 2011, 10, 155-162.

24. Muleya T., Rolny V., He Y., Wehnl B., Escherich A., Warth A., Stolp C., Schneider M.A., Dienemann H., Meister M., Herth F.J., Dayyani F.: The combination of the blood based tumor biomarkers cytokeratin 19 fragments (CYFRA 21-1) and carcinoembryonic antigen (CEA) as a potential predictor of benefit from adjuvant chemotherapy in early stage squamous cell carcinoma of the lung (SCC). Lung Cancer 2018, 120, 46-53.

25. Nguyen A.H., Miller E.J., Wichman C.S., Berim I.G., Agrawal D.K.: Diagnostic value of tumor antigens in malignant pleural effusion: a meta-analysis. Transl Res 2015, 166, 433-439.

26. Nikolouzakis T.K., Vassilopoulou L., Fragkiadaki P., Sapsakos T.M., Papadakis G.Z., Spandidos D.A.: Improving diagnosis, prognosis, and prediction by using biomarkers in CRC patients (Review). Oncol Rep 2018, 39, 2455-2472. 
27. Nisbet D.I., Mackay J.M., Smith W., Gray E.W.: Ultrastructure of sheep pulmonary adenomatosis (Jaagsiekte). J Pathol 1971, 103,157-162.

28. Palmarini M., Cousens C., Dalziel R.G., Bai J., Stedman K., DeMartini J.C., Sharp J.M.: The exogenous form of jaagsiekte retrovirus is specifically associated with a contagious lung cancer of sheep. J Virol 1996, 70, 1618-1623.

29. Radad K., Khalil S.: Natural ovine pulmonary adenocarcinoma in an Egyptian sheep farm. Eurasian J Vet Sci 2014, 30, 39-43.

30. Salgia R., Harpole D., Herndon J.A., Pisick E., Elias A., Skarin A.T.: Role of serum tumor markers CA 125 and CEA in non-small cell lung cancer. Anticancer Res 2001, 21, 1241-1246.

31. Scott P.R.: Sheep medicine. CRC press, Boca Raton, 2015.

32. Sonawane G.G., Tripathi B.N., Kumar R., Kumar J.: Diagnosis and prevalence of ovine pulmonary adenocarcinoma in lung tissues of naturally infected farm sheep. Vet World 2016, 9, 365-370, doi:10.14202/vetworld.2016.365-370.

33. Sozmen M., Beytut E.: An investigation of growth factors and lactoferrin in naturally occurring ovine pulmonary adenomatosis. J Comp Pathol 2012, 147, 441-451.

34. Tang H., Bai Y., Shen W., Wei Y., Xu M., Zhou X.: Clinical significance of combined detection of interleukin- 6 and tumour markers in lung cancer. Autoimmunity 2018, 51, 191-198.

35. Tomita M., Matsuzaki Y., Edagawa M., Shimizu T., Hara M., Onitsuka T.: Prognostic significance of preoperative serum carcinoembryonic antigen level in lung adenocarcinoma but not squamous cell carcinoma. Ann Thorac Cardiovasc Surg 2004, 10, $76-80$
36. Tsoukalas N., Kostakis I.D., Giaginis C., Tolia M., Galanopoulos M., Kiakou M., Aravantinou-Fatorou E., Tsapakidis K., Baxevanos P., Litos I., Tzouda V., Tzovaras A., Kyrgias G., Tsiambas E. Theocharis S.: carcinoembryonic antigen and carbohydrate antigen 19-9 serum levels in non-small cell lung cancer. J Buon 2017, 22, 1390-1394.

37. Tuxen M.K., Soletormos G., Dombernowsky P.: Tumor markers in the management of patients with ovarian cancer. Cancer Treat Rev 1995, 21, 215-243.

38. Wang W.J., Tao Z., Gu W., Sun L.H.: Clinical observations on the association between diagnosis of lung cancer and serum tumor markers in combination. Asian Pac J Cancer Prev 2013, 14, 4369-4371.

39. Xu-Welliver M., Carbone D.P.: Blood-based biomarkers in lung cancer: prognosis and treatment decisions. Transl Lung Cancer Res 2017, 6, 708-712.

40. Youssef G., Wallace W.A.H., Dagleish M.P., Cousens C., Griffiths D.J.: Ovine pulmonary adenocarcinoma: a large animal model for human lung cancer. ILAR J 2015, 56, 99-115.

41. Yu M., Wang Y., Abdalla A.M.E., Liu W., Mei F., Wang J., Ouyang C., Li Y.: Carbohydrate antigens as potential biomarkers for the malignancy in patients with idiopathic deep venous thrombosis: a retrospective cohort study. J Huazhong Univ Sci Technolog 2014, 34, 722-728

42. Zamay T.N., Zamay G.S., Kolovskaya O.S., Zukov R.A., Petrova M.M., Gargaun A., Berezovski M.V., Kichkailo A.S.: Current and prospective protein biomarkers of lung cancer. Cancers 2017, 9, 1-22. 